\title{
CUERPO Y ALMA EN EL HILEMORFISMO DE SANTO TOMÁS
}

\author{
Mauricio Beuchot \\ UNAM (México)
}

\section{INTRODUCCIÓN}

El presente trabajo tiene por cometido presentar algunos elementos de la doctrina de Santo Tomás de Aquino acerca del alma humana. Primero recordaremos su herencia aristotélica, que es el hilemorfismo, la teoría del compuesto materia/forma aplicada al hombre. Después analizaremos algunos argumentos que intentan probar que el alma es distinta del cuerpo e irreductible a algo material. Aprovecharemos la argumentación de Anthony Kenny y de Norman Malcolm, para apoyar desde diversas perspectivas la de Santo Tomás. Después de hablar acerca de la existencia del alma, pasaremos a su esencia no naturaleza; de ella abordaremos el problema de si es una o múltiple, esto es, si, ya que en el hombre hay un aspecto vegetativo, otro sensitivo y otro racional, hay en él tres almas o una sola. Otro problema tocante a la naturaleza del alma es el de su origen en el cuerpo 0 , si se prefiere, en el compuesto humano.

En el sistema de Santo Tomás, el hombre es un compuesto hilemórfico de cuerpo y alma. En esto sigue fundamentalmente a Aristóteles, pero difiere de él, ya que toma en cuenta la doctrina cristiana de la inmortalidad del alma -inmortalidad que en el Estagirita nunca estuvo del todo clara- y la ampliación de la diada materia-forma a esencia-existencia -que es una aportación de Santo Tomás-. Todo eso tendrá, como veremos, sus consecuencias.

\section{EL HILEMORFISMO ARISTOTÉLICO Y TOMISTA}

El compuesto hilemórfico consta de materia prima y forma substancial (ambas son lo más elemental: la materia primera y la forma primera). Por eso cuando aludimos al cuerpo, éste debe entenderse como la materia prima del compuesto humano, esto es, el receptáculo o sujeto o substrato; y el alma racional o espiritual como al forma substancial o primera de dicho 
compuesto. En ese compuesto el cuerpo y el alma se unen substancialmente, como lo hacen en el hilemorfismo la materia y la forma, que juntos constituyen la substancia. Si no se unieran substancialmente, lo harían accidentalmente, con lo cual serían inconciliables. Por tanto, la postura tomista no es monista, en la que sólo habría cuerpo o sólo alma, no es ni materialismo ni espiritualismo; es una postura dualista: dos cosas se unen entre sí; pero dicha unión tiene que ser substancial o accidental; si es substancial, ya no siguen siendo sin más dos cosas, sino en cierto modo una cosa con dos aspectos, se trata de un dualismo sui generis; $y$, si es accidental, son dos cosas que en verdad están irreconciliablemente separadas, la una junto a la otra solamente. En cambio, en la unión substancial, sin dejar de ser cada cosa lo que es, se compenetran de manera tal que casi son una sola cosa. Por así decir, Santo Tomás fue lo menos dualista y lo más monista que se podía ser en el cristianismo.

Para comprender la relación entre el alma y cuerpo, hemos de entender bien el status ontológico de ambos correlatos y el modo de la unión. Para ello habrá que recordar algunas nociones del hilemorfismo aristotélico; luego analizar la posibilidad de la existencia misma del alma, esto es, de su no reductibilidad al cuerpo, a la materia; y finalmente examinar algunos aspectos de su estructura o naturaleza.

El sistema de filosofía natural sostenido por Aristóteles y Santo Tomás se llama hilemorfismo porque indica que la substancia individual se compone de materia (hyle) y forma (morphé). Aristóteles supuso que, para explicar el movimiento, tenía que dar razón principalmente del cambio substancial. En él, algo de la cosa cambiaba y algo quedaba. Por ejemplo, el leño se quemaba, algo se perdía en la transformación pero algo quedaba: el carbón. Postuló, pues, un principio de permanencia: la materia prima, y un principio de cambio: la forma primera. La permanencia se explicaba porque quedaba el mismo substrato: la materia, pero había cambio porque la forma anterior de la cosa había desaparecido dejando lugar a la nueva forma: se fue la forma de leño y quedó la forma de carbón. Las substancias individuales, entonces, tienen una materia y una forma. Esa era la diada aristotélica: materia y forma, la primera es la parte potencial y la segunda la parte actual o de acto.

Santo Tomás añadió otra diada más fundamental en el ente: la esencia y la existencia o ser. La esencia comprende la materia y la forma y la existencia o ser (esse) completa a la esencia, la perfecciona y le da actualidad como cosa existente. Ese acto de ser viene a la substancia no por parte de la materia, sino por parte de la forma. Decían los escolásticos: «forma dat esse rei» ( «la forma da el ser a la cosa»), porque la forma es la que actualiza a la materia, y a través de la forma se recibe el ser en ese todo hilemórfico que es la esencia de la cosa, resultando entonces la cosa o substancia existente. Ya que son principios de la substancia, la materia prima puede considerarse como materia substancial y la forma prima como forma substancial. De su composición resulta la substancia completa, y no pueden ser substancias completas cada una de ellas. La materia no puede subsistir sola, sin la forma, pues es potencia pura, está necesitada de la forma: pero la forma puede en ocasiones subsistir sin materia, como en el caso de las formas separadas. Uno de estos casos será el del alma, que es una substancia incompleta dentro de una substancia completa que es el compuesto humano. Es ciertamente un compuesto muy 
peculiar. Mas, por ahora, dejando para otra parte la discusión de esa composición tan peculiar en la que el alma se une al cuerpo como una substancia incompleta, examinemos la composición del alma con el cuerpo como la forma con la materia, composición en la que el alma es el principio vital o principio de movimiento o acto primero del cuerpo orgánico.

\section{ARGUMENTACIÓN TENDENTE A PROBAR LA EXISTENCIA DE UN ALMA IRREDUCTIBLE AL CUERPO}

Anthony Kenny hace ver que se puede usar la noción de alma (en sentido aristotélico: principio vital o de automovimiento), si no se confunde con la noción de mente; la mente es sólo una parte suya; pero, en el caso del hombre se puede hablar de «alma intelectual» como equivalente a «mente». Así, la mente sería sólo una parte del alma, la que se encarga de las actividades cognoscitivas y volitivas, mientras que el alma, en este sentido más amplio, puede definirse simplemente como el principio de toda actividad del organismo. Kenny define la mente como «la capacidad de adquirir habilidades intelectuales». ${ }^{1}$

De otro modo parecido, la mente puede describirse así: «En su sentido primario, la mente humana es la capacidad de adquirir habilidades intelectuales. Es una capacidad, no una actividad: los bebés tienen mentes aun cuando no exiban aún actividades intelectuales. Es una capacidad de segundo orden: una habilidad de adquirir o poseer habilidades. Saber un lenguaje es tener una habilidad: la habilidad de hablar, entender y quizá leer el lenguaje. Tener una mente es tener una capacidad un peldaño más abajo: la habilidad de adquirir habilidades tales como el conocimiento de un lenguaje». ${ }^{2}$

Es, pues una capacidad de segundo orden, capacidad de capacidades.

Muchos errores vienen de no ver la mente como una capacidad, sino como una actividad (conductismo), es decir, de no verla como capacidad de segundo orden, sino como ejercicios particulares en la conducta; otros errores vienen de reducir las capacidades mentales a las partes y aspectos estructurales del cuerpo, por las que tenemos esas capacidades (materialismo). ${ }^{3}$ Ambos son reduccionismos indebidos. No se puede confundir un cuerpo con sus capacidades. Kenny añade que tanto el dualismo (cartesiano) como el conductismo quieren poner en duda cosas que todos sabemos que son verdaderas. El conductismo pone en duda que tengamos pensamientos o sentimientos que no se exiban de manera pública; y sabemos que eso es falso. $O$ que mi conciencia de pensamientos y sentimientos es una inferencia a partir de hipótesis sobre mi conducta manifiesta; lo cual es absurdo. El dualismo cartesiano conduce al escepticismo sobre otras mentes, sólo vale la introspección, que es individual, mía. Ni siquiera permite una deducción causal a partir del comportamiento de los otros hacia sus conciencias

1 Kenny, A., «The Origin of the Soul», en Varios, The Development o Mind, Edinburg: University Press, 1973, p. 46.

2 Loem, The Metaphysics of Mind, Oxford: Clarendon Press, 1989, p. 20.

3 Cfr. Idem, «The Origin of the Soul», ed. cit., p. 51. 
(porque uno de los términos de la relación es inobservable). Pero eso es tan reductivo que resulta ridículo. Por ello Kenny se alegra de que, afortunadamente, no son las únicas alternativas; dice que el mejor filósofo de la mente en este siglo ha sido Wittgenstein, y él se colocaba a medio camino de ambos. Según él, unos y otros fueron víctimas de una confusión. Los eventos mentales ni son reductibles a expresiones corpóreas (materialismo conductista) ni completamente separables de ellas (dualismo cartesiano). Incluso lo más privado lo hacemos con el lenguaje, que está esencialmente vinculado a las expresiones corpóreas públicas. Kenny hace ver que no son lo mismo criterio y síntoma. Las descargas eléctricas en el cerebro son sólo síntomas de cierta competencia en un idioma; pero el criterio es la ejecución o el uso del idioma. ${ }^{4}$ Los conceptos mentalistas (deseo, creencia, intención, motivo y razón) sirven para explicar la conducta humana, pero no son una teoría que extraemos a partir de la conducta, de modo hipotético y causal. Sólo es para simplificar, para entender e interpretar dicha conducta. ${ }^{5}$

Así pues, la reflexión de Kenny nos sirve para ver la coherencia y la adecuación de las nociones empleadas por Sto. Tomás en su construcción doctrinal. Además, nos sirve para ver que lo mental o anímico no es de ninguna manera reductible a lo corpóreo. En esta misma línea de tal irreductibilidad se sitúan las consideraciones de Norman Malcolm, que aprovecharemos para reforzar esa tesis.

También Malcolm defiende, con otros argumentos, la irreductibilidad de la mente al cuerpo. Combate al menos el materialismo en dos de sus proponentes, que son J. J.C. Smart y U. T. Place. ${ }^{6}$ El materialismo (o monismo materialista) tiene que sostener cierta identidad entre procesos mentales y procesos cerebrales. Pero esa identidad no puede ser lógicamente necesaria (o, como dice Smart, «estricta»); porque de otra manera, si fuera lógicamente necesaria, un evento mental tendría el mismo espacio y tiempo que un evento cerebral, y por lo tanto un estado mental tendría verificación empírica, al igual que el estado cerebral; lo cual no es comprensible. Sólo puede ser, por ello, una identidad contingente. ${ }^{7}$ Es decir, si se quisiera

4 Cfr. Idem, The Metaphysics of Mind, ed. cit., p. 5.

5 Kenny dice que no hace falta entrar en debate acerca de la existencia de almas sin cuerpo entendidas como personas, pues el propio Sto. Tomás creía que sólo eran propiamente personas las almas con su cuerpo: «No deseo, sin embargo, debatir con los que creen en almas inmortales descorporeizadas. Pues lo que acabo de decir no sería necesariamente rechazado por aquéllos que creen en tales almas. Podemos tomar a Sto. Tomás de Aquino como un vocero de tales creyentes. El Aquinate sin duda creyó que cada ser humano tenía un alma inmortal, que podría sobrevivir a la muerte del cuerpo y continuar queriendo y pensando en el periodo antes de la eventual resurrección del cuerpo que esperaba. Con todo, el Aquinate no creía que podía sobrevivir, como la persona que era, en un yo que fuera distinto del cuerpo, porque no pensaba que las almas descorporeizadas fueran personas. Aun después de la muerte, creía que su alma era el alma que era sólo porque era el alma que había sido el alma de un cuerpo particular. La supervivencia personal plena, según él, era posible sólo si hubiera una resurrección del cuerpo» (Ibid., p. 31)

6 Cfr. Smart, J.J.C., «Sensations and Brain Processes», en Philosphical Review, 68. 1959; Idem, «Materialism», en Journal of Philosophy, 60 (1963); U. T. Place, «Is Consciousness a Brain Process», en British Journal of Psychology, 47. 1956.

7 Cfr. Malcolm, N., Problems of Mind. From Descartes to Wittgenstein, New York: Harper and Row, 1971, p. 65 . 
que el proceso mental y el proceso cerebral tuvieran una identidad lógica o estricta, se tendría que cumplir la siguiente condición necesaria: «Si $x$ ocurre en un cierto lugar en cierto tiempo, entonces $y$ es estrictamente idéntica a $x$ si y sólo si $y$ ocurre en el mismo lugar al mismo tiempo». ${ }^{8}$ Lo cual no parece estar dispuesto a decir el materialista. Por ejemplo -arguye MalcolmSmart cree que un relámpago es "estrictamente idéntico» a una descarga eléctrica, de modo que cuando ocurre un relámpago ocurre una descarga en el mismo lugar y tiempo; pero no hará así idénticos los estados mentales y las descargas eléctricas cerebrales. Tampoco Place lo aceptaría así.

En efecto, explica Malcolm, un proceso cerebral ocurre en cierto tiempo y locación. Es un proceso eléctrico, mecánico, químico, o físico, etc. Y puede verificarse mediante instrumentos científicos. Pero sólo se identifica con un proceso mental si puede verificarse que este último ocurrió en el cerebro, dentro del cráneo. Pero ¿puede hacerse? Malcolm responde:«Debe notarse que, en cuanto la identidad se presume de ser contingente, el método para determinar que un pensamiento o un pensar está localizado dentro del cráneo tendría que ser lógicamente independiente del método para determinar la ocurrencia del proceso cerebral correlativo. Emplear la ocurrencia del proceso cerebral como el criterio de la ocurrencia del pensamiento dentro del cráneo prevendría a la identidad putativa para tener una verificación empírica. Ya no sería más concebida como una identidad contingente. Habría una conexión de significado entre decir que un cierto proceso cerebral ocurrió dentro del cráneo de alguien y decir que un cierto pensamiento ocurrió en el mismo lugar». ${ }^{9}$ Mas, si se ha de conservar la identidad como contingente y la verificación como empírica -que es a lo que se llegó por los razonamientos anteriores-, no hay manera de investigar -ni por observación ni con instrumentos- la ocurrencia de un pensamiento dentro del cráneo.

Sólo podemos atender a ciertos efectos (expresiones, acciones, gestos, etc.) para determinar estados mentales. Pero esto es conceptualmente independiente de la investigación de procesos cerebrales. Nunca nos dirá nada acerca de alguna locación corpórea de ese evento mental. No sabríamos qué significa eso de que un pensamiento esté localizado, digamos, en el cerebro. Ni siquiera entenderíamos lo que eso quiere decir. ${ }^{10} \mathrm{Y}$ si se le da un significado convencional, o convenido, será una definición de lo que significa; pero la identidad que surgió de esa convención seguirá siendo contingente, porque habría sido verificada sólo en parte, asignando un sentido a una expresión que carecía de él.

Smart llegó a denunciar que lo que pasaba era que en el lenguaje ordinario hay una tendencia al dualismo metafísico, y que por eso no tenían sentido las expresiones que él defendía; pero que, si se hiciera una revisión conceptual y se colocaran las experiencias conscientes en la cabeza, habría una correlación acorde con la ciencia de hoy. A eso contesta Malcolm que ciertamente los cambios conceptuales y lingüísticos ocurren pero cuando hay un sentido que los respalde. Antes no tenía sentido asignar valores numéricos a la temperatura;

8 Ibid., p. 66

9 Ibid., p. 67

10 Sobre esto Malcolm cita el brillante estudio de J.A. Shaffer, «Recent Work on the Mind-Body Problem», en American Philosophical Quarterly, 2 (1965), p. 97. 
hubo que inventar el termómetro para que lo tuviera. Si se pudieran correlacionar los eventos fisiológicos con los mentales de modo que hubiera un criterio de lo que es la locación de un pensamiento, entonces ese cambio tendría sentido. Pero si se trata de una identidad entre ellos, no admitiría comprobación ni disprobación empírica. Malcolm reflexiona: «la confesión de Smart de que a causa del sesgo del lenguaje ordinario hacia una "metafísica dualista" se requiere una revisión del lenguaje para establecer la conjetura de la identidad como verdadera es una admisión de que no hay posibilidad lógica de que la conjetura sea establecida como contingentemente verdadera o como contingentemente falsa». ${ }^{11}$ Es decir, no se puede dar la identidad necesaria, estricta o lógica entre mente y cuerpo; sólo puede ser contingente; pues, si no, los fenómenos mentales ocuparían el mismo lugar y tiempo que los cerebrales; pero esto de que los pensamientos ocupen un lugar no tiene sentido; $y$, de esta manera, sólo tienen identidad contingente; pero si esto es así, ya no se pueden investigar por el mismo método científico, de modo que sean verificadas empíricamente.

\section{UNIDAD O PLURALIDAD DE FORMAS EN EL COMPUESTO HUMANO}

Algunos pensadores habían dicho que la forma substancial el hombre era múltiple, no unitaria. Es decir, que en el hombre, ya que tiene un aspecto vegetativo, otro sensitivo y otro racional, había tres almas, correspondientes a esas tres dimensiones. Y eso hacía que su forma substancial fuera múltiple. Pero Santo Tomás dice que más bien es una sola alma, que, por ser más perfecta, puede hacer las funciones de las otras dos, pues lo que puede lo más puede lo menos; y, así, no es necesario que el hombre tenga alma racional y además alma sensitiva y alma vegetativa, sino que su alma racional alcanzara a cumplir lo de las otras. Por eso dice: «No existe en el hombre otra forma substancial fuera del alma intelectiva, alma que virtualmente contiene la forma sensitiva y vegetativa, así como también contiene todas las demás formas interiores, ya que ella sola puede hacer todo lo que las formas menos perfectas que ella pueden hacer».12 Niega, pues, la pluralidad de las formas, ya que la sola alma racional tiene virtualmente a las otras dos, es decir, no real, actual o formalmente, sino en cuanto a las operaciones o potencias que ellas desarrollan: «Por su forma substancial, que es humana, el hombre no solamente es hombre, sino también animal, planta, cuerpo, substancia y ser». ${ }^{13} \mathrm{~A}$ lo cual añade: «La forma más perfecta hace de modo unitario todas las cosas que las inferiores hacen de modo diversificado, y aún más: por ejemplo, si la forma del cuerpo inanimado da a la materia el ser y el ser cuerpo, la forma de la planta le dará eso y además el vivir; y el alma sensitiva eso y además el ser sensible; y el alma racional, eso y además el ser racional». ${ }^{14} \mathrm{O}$, como dice en otro lugar el propio Sto. Tomás: «No existe sino un ser substancial en cada

11 Ibid., p. 72.

12 Sum. Theol., I, q. 76 a. 4.

13 De spiritualibus creaturis, a.3.

14 De spiritualibus creaturis, a. 4; cfr. In II De An., lect. 1, n. 234. 
substancia. La forma substancial es, pues, la que da a una cosa su ser substancial. Por tanto, cada cosa no posee sino una forma substancial, y puesto que el alma es la forma substancial del hombre, síguese que no puede haber en él otra forma substancial que no sea el alma intelectual». ${ }^{15} \mathrm{Y}$, si tuviera un alma vegetativa, otra sensitiva y otra racional, el hombre no sería uno y simple. Sólo puede tener el alma sensitiva y vegetativa virtual o potencialmente en la racional; pero no tiene igual que el animal su vida sensitiva ni igual que la planta su vida vegetativa; solamente de modo análogo a ellos. ${ }^{16}$

\section{APOSTILLA SOBRE EL ORIGEN DEL ALMA}

Por último, en cuanto al origen del alma, Santo Tomás piensa que no se transmite por parte de los padres, sino que es creada por Dios para cada nuevo ser humano que se produce. Kenny apoya en esto a Santo Tomás y alude a la mayor coherencia de ello con el dictum escolástico «forma nec est nec fit nec generatur» ${ }^{17}$ (la forma ni existe ni se hace ni se genera). No existe, porque no tiene existencia por si sola, sino que es en el compuesto con la materia -a menos que sea una forma pura, como Dios o los ángeles, de quienes no se trata aquí-; tampoco se hace en el sentido físico, sino espiritual, por creación divina; ni se genera, en el sentido de que no es engendrada por los padres. San Agustín -recuerda Kenny- decía que el alma se heredaba de los padres; San Jerónimo que era creada en cada uno por Dios. A Kenny le parece más razonable lo de San Jerónimo: cada ser humano es algo nuevo y totalmente inédito, inexplicable por la historia hereditaria de algo. Aun cuando no se acepte la existencia de Dios, habría que pensar el alma no como algo transmitido como herencia biológica, sino como algo que surgió.

Y no se incurre en la falacia del homúnculo, pues no se dice que el cuerpo esté habitado por un alma como hombrecillo interior, sino que la persona es un cuerpo que tiene ciertas capacidades que constituyen la mente.

\section{CONCLUSIÓN}

Hemos visto, conducidos por Kenny y Malcolm, cómo la noción de alma en cuanto distinta del cuerpo tiene sentido y es defendible en contra de los monistas materialistas, que vienen a quedar como reduccionistas. La noción de alma es irreductible a la de cuerpo, y además el lenguaje del alma ayuda a la comprensión de los fenómenos humanos sin que sea factible prescindir de él.

También hemos visto que la doctrina del alma dada por Santo Tomás -dentro de su contexto hilemorfista-, es adecuada para explicar su naturaleza, su génesis y su unicidad. No

15 Sum. Theol., I, q. 76, a.4.

16 Cfr. Sum. Theol., I, q: 76, a.3.

17 Cfr. Kenny, A., The Metaphysics of the Mind, ed. cit., p. 54 
es reduccionista como el monismo materialista o el espiritualista; mantiene un dualismo en el que, sin embargo, trata de conjuntar al máximo ambos elementos del compuesto. Trata de conectar el alma con el cuerpo, procurando evitar el otro extremo que es el dualismo cartesiano, en el que es sumamente difícil explicar su interacción. Da la mejor explicación del compuesto humano, con una sola materia y una sola forma (no pluralidad de formas, que habría si hubiera muchas almas). También explica el origen del alma de la manera más sencilla y clara, no como transmitida por los progenitores, sino por creación directa de Dios. Todo eso nos habla de lo consistente y adecuada que es la filosofía tomista del hombre. 